\title{
Le Cid (I, 1-2, 1-52) de Pierre Corneille
}

Traduction et note à la traduction

\section{Angela Di Benedetto}

\section{(2) OpenEdition}

Journals

Édition électronique

URL : http://journals.openedition.org/rief/275

DOI : 10.4000/rief.275

ISSN : 2240-7456

Éditeur

Seminario di filologia francese

\section{Référence électronique}

Angela Di Benedetto, «Le Cid (I, 1-2, 1-52) de Pierre Corneille », Revue italienne d'études françaises [En ligne], 3 | 2013, mis en ligne le 15 décembre 2013, consulté le 22 septembre 2020. URL : http:// journals.openedition.org/rief/275; DOI : https://doi.org/10.4000/rief.275

Ce document a été généré automatiquement le 22 septembre 2020.

\section{(c) (i)}

Les contenus de la RIEF sont mis à disposition selon les termes de la Licence Creative Commons Attribution - Pas d'Utilisation Commerciale - Pas de Modification 4.0 International. 


\title{
Le Cid (I, 1-2, 1-52) de Pierre Corneille
}

Traduction et note à la traduction

\section{Angela Di Benedetto}

\section{RÉFÉRENCE}

Le Cid (1637), I, 1-2, 1-52, dans Euvres Complètes, éd. G. Couton, Paris, Gallimard, «Bibliothèque de la Pléiade », vol. I, 1980, p. 709-711

\section{Version originale}

\author{
ACTE PREMIER \\ Scène première \\ LE COMTE, ELVIRE
}

ELVIRE

Entre tous ces amants dont la jeune ferveur

Adore votre fille, et brigue ma faveur,

Don Rodrigue et Don Sanche à l'envi font paraître

Le beau feu qu'en leurs cœurs ses beautés ont fait naître.

Ce n'est pas que Chimène écoute leurs soupirs, 5

Ou d'un regard propice anime leurs désirs.

Au contraire, pour tous dedans l'indifférence

Elle n'ôte à pas un, ni donne d'espérance,

Et sans les voir d'un œil trop sévère, ou trop doux,

C'est de votre seul choix qu'elle attend un époux. 10

LE COMTE

Elle est dans le devoir ; tous deux sont dignes d'elle, Tous deux formés d'un sang noble, vaillant, fidèle, 
Jeunes, mais qui font lire aisément dans leurs yeux L'éclatante vertu de leurs braves aïeux.

Don Rodrigue surtout n'a trait en son visage 15 Qui d'un homme de cœur ne soit la haute image, Et sort d'une maison si féconde en guerriers Qu'ils y prennent naissance au milieu des lauriers. La valeur de son père, en son temps sans pareille, Tant qu'a duré sa force, a passé pour merveille, 20 Ses rides sur son front ont gravé ses exploits, Et nous disent encor ce qu'il fut autrefois : Je me promets du fils ce que j'ai vu du père, Et ma fille en un mot peut l'aimer et me plaire. Va l'en entretenir, mais dans cet entretien, 25 Cache mon sentiment et découvre le sien.

Je veux qu'à mon retour nous en parlions ensemble ; L'heure à présent m'appelle au conseil qui s'assemble, Le Roi doit à son fils choisir un Gouverneur, Ou plutôt m'élever à ce haut rang d'honneur. 30 Ce que pour lui mon bras chaque jour exécute, Me défend de penser qu'aucun me le dispute.

Scène seconde

CHIMÈNE, ELVIRE

ELVIRE, seule.

Quelle douce nouvelle à ces jeunes amants !

Et que tout se dispose à leurs contentements ! CHIMÈNE

Eh bien, Elvire, enfin, que faut-il que j'espère ? 35

Que dois-je devenir, et que t'a dit mon père?

ELVIRE

Deux mots dont tous vos sens doivent être charmés.

Il estime Rodrigue autant que vous l'aimez.

CHIMÈNE

L'excès de ce bonheur me met en défiance, Puis-je à de tels discours donner quelque croyance ? 40 ELVIRE

Il passe bien plus outre, il approuve ses feux, Et vous doit commander de répondre à ses vœux. Jugez après cela puisque tantôt son père Au sortir du Conseil doit proposer l'affaire, S'il pouvait avoir lieu de mieux prendre son temps, 45 Et si tous vos désirs seront bientôt contents. CHIMÈNE

Il semble toutefois que mon âme troublée Refuse cette joie, et s'en trouve accablée, Un moment donne au sort des visages divers, Et dans ce grand bonheur je crains un grand revers. 50 ELVIRE

Vous verrez votre crainte heureusement déçue. 
CHIMÈNE

Allons, quoi qu'il en soit, en attendre l'issue.

\section{Traduction italienne}

ATTO PRIMO

Scena prima

IL CONTE, ELVIRA

ELVIRA

Tra tutti quei giovani e fervidi pretendenti

Che adorano vostra figlia e brigano il mio favore, Don Rodrigo e Don Sancio fanno apparire a gara Il fuoco che in cuor loro la sua bellezza accende. Di certo Chimène non ne ascolta i sospiri, 5 Né con occhio propizio ne incoraggia i desideri. Al contrario, sempre indifferente verso ognuno, Non toglie, né concede speranza ad alcuno.

E senza guardarli in modo troppo dolce o duro, Attende il suo sposo solo dalla vostra scelta. 10 IL CONTE

Fa il suo dovere ; entrambi sono degni di lei, Forgiati da sangue nobile, audace, leale, Giovani, ma i cui occhi rivelano chiaramente L'eclatante virtù di antenati valorosi. In specie Don Rodrigo non ha tratto del viso 15 Che non riveli il carattere d'un uomo ardito, Viene da un casato così fecondo in guerrieri, Che vi vengono al mondo già in mezzo agli allori. Il valore del padre, ai suoi tempi senza eguale, Finché aveva forza, ha suscitato meraviglia ; 20 Le sue rughe hanno incise in fronte le imprese, E ci dicono ancora quel che in altri tempi fu. Aspetto dal figlio quel che ho visto del padre ; Mia figlia, in una parola, può amarlo e piacermi. Va a parlarle, ma nel vostro colloquio 25 Nascondi la mia inclinazione e scopri la sua. Quando torno voglio che ne parliamo assieme ; Il Consiglio si riunisce, adesso devo andare. Il Re deve scegliere un Governatore al figlio $O$ piuttosto innalzarmi a questo rango d'onore. 30 Ogni giorno quanto il mio braccio compie per lui M'impedisce di credere che mi sia conteso.

Scena seconda

CHIMÈNE, ELVIRA

ELVIRA, sola.

Che dolce notizia per questi giovani amanti !

Tutto si aggiusta secondo il loro desiderio! 
CHIMÈNE

Ebbene Elvira, insomma, cosa devo sperare ? 35

Cosa ne sarà di me ? Che ha detto mio padre?

ELVIRA

Due parole che vi devono sciogliere tutta :

Stima tanto Rodrigo quanto voi l'amate.

CHIMÈNE

La troppa felicità m'induce a diffidare :

Che credito posso accordare a tali discorsi ? 40

ELVIRA

Si spinge ancor più in là, approva il suo ardore

E vi ordina di rispondere ai suoi sentimenti.

Da questo giudicate, giacché ora suo padre

Deve proporre l'affare uscendo dal Consiglio,

Se poteva trovare un'occasione migliore, 45

E se sarà presto accolto il vostro desiderio.

CHIMÈNE

Sembra tuttavia che il mio animo turbato

Rifiuti questa gioia, e se ne senta oppresso.

In un istante la sorte può cambiare volto

$E$ in tanta felicità temo un gran rovescio. 50

ELVIRA

La vostra paura sarà felicemente vinta.

CHIMÈNE

Qualunque cosa sia, attendiamone l'esito.

\section{Note à la traduction}

1 J'ai choisi de traduire les deux premières scènes de la tragi-comédie dans la version conforme à la première édition Picot 1637, telle que l'a reproduite Georges Couton. La traduction du Cid par Guido Davico Bonino (Einaudi, 1999), la plus importante actuellement encore dans les librairies italiennes, est réalisée sur le texte de la dernière édition, celle de 1682. Je présente donc ici la première scène, qui fut supprimée à partir de l'édition 1660, et la deuxième, devenue la première, et profondément modifiée. À partir de l'édition 1648, la tragi-comédie a été désignée comme tragédie par l'auteur.

2 À la différence des nombreuses variantes de la première édition, principalement dictées par des raisons de vraisemblance et de conformité au nouveau goût classique, ce sont des motivations strictement dramaturgiques qui sont à l'origine de la substitution de cette première scène. Dans la version définitive, la rencontre d'Elvire avec le Comte est éliminée : elle n'est que racontée à Chimène. Une répétition est ainsi abolie ; et surtout, et c'est là l'important, le spectateur apprend en même temps que son père est favorable au prétendant pour qui penche son cœur. Une scène d'exposition se voit ainsi investie de l'affectivité du personnage qui l'écoute.

3 En ce qui concerne la versification, j'ai choisi de ne pas rendre l'alexandrin par le martelliano avec césure fixe entre deux heptasyllabes ; j'ai préféré un vers de 14 pieds à coupes irrégulières, à la façon de l'hendécasyllabe italien. Le résultat de cette 
versification, avec l'abolition presque totale de la rime, devrait être une sorte de prose rythmée.

4 Pour le lexique, j'ai essayé de respecter le style non seulement des personnages mais aussi celui que le rang des interlocuteurs et les circonstances imposent (l'aptum cicéronien que l'honnête homme du XVII ${ }^{e}$ siècle avait fait sien). Ainsi j'ai traduit «amants» (v. 1), terme employé par Elvire, par «pretendenti» à la place du plus sentimental «innamorati» ou du plus mondain "corteggiatori», parce qu'une connotation plus officielle était conforme à la dignité paternelle du Comte auquel elle parle. Mais quand Elvire s'adresse à Chimène, elle change de registre stylistique. J'ai donc traduit le vers 34 « Tout se dispose à leurs contentements » par « Tutto si aggiusta secondo il loro desiderio!» et le vers 37 « Deux mots dont tous vos sens doivent être charmés " par "Due parole che vi devono sciogliere tutta", expression qui contient une allusion à la tendre sensibilité de Chimène.

5 Pour ce qui concerne le lexique galant, j'ai traduit "vœux » par « sentimenti », tandis que j'ai employé pour «feu » une fois « fuoco », et la seconde fois " ardore » parce qu'il y a dans cette tragi-comédie un motif évoquant la chaleur et la lumière («ferveur, éclatante») qui s'oppose au froid; j'ai renforcé ce motif, au vers 4, par le verbe « accende », pour le français « fait naître ».

6 Quant à la syntaxe, j'ai essayé de respecter la structure de la phrase française et de conserver dans chaque vers la construction originale des phrases; à l'exception des vers $1-2$, où le remplacement du nom «ferveur » par l'adjectif «fervidi », précédant le substantif " pretendenti », a modifié la structure des relatives qui dépendaient du nom ferveur ( entre tous ces amants dont la jeune ferveur adore votre fille et brigue ma faveur " / "fra tutti quei giovani e fervidi pretendenti che adorano vostra figlia e brigano il mio favore »); et au vers 28 , où j'ai préféré scinder la période "l'heure à présent m'appelle au conseil qui s'assemble ", en deux phrases simples, qui deviennent ainsi deux propositions indépendantes - «Il Consiglio si riunisce, adesso devo andare »- pour rendre plus naturelle la sortie de scène du Comte et en motiver l'urgence.

\section{INDEX}

Mots-clés : traduction, vers classique, Cid, Corneille (Pierre), alexandrin, martelliano 